\title{
Bifurcações Sela-Nó da Região de Estabilidade de Sistemas Dinâmicos Autônomos não Lineares
}

\author{
F.M. AMARAL ${ }^{1 *}$ e L.F.C. ALBERTO ${ }^{2}$ \\ Recebido em 17 maio, 2015 / Aceito em 15 janeiro, 2016
}

\begin{abstract}
RESUMO. O comportamento da região de estabilidade de sistemas dinâmicos sujeitos a variação de parâmetros é estudado neste artigo. O comportamento da região de estabilidade e de sua fronteira quando o sistema vai de encontro a uma bifurcação sela-nó do tipo- $k$, com $k \geq 0$ na fronteira da região de estabilidade é investigado. Uma caracterização completa da fronteira da região de estabilidade na vizinhança de um valor de bifurcação sela-nó do tipo- $k, \operatorname{com} k \geq 0$ é apresentado neste artigo.
\end{abstract}

Palavras-chave: região de estabilidade, fronteira da região de estabilidade, bifurcação sela-nó.

\section{INTRODUÇÃO}

Caracterizações dinâmicas e topológicas da fronteira da região de estabilidade de sistemas dinâmicos autônomos não lineares podem ser encontradas, por exemplo em [4] e [9]. As caracterizações existentes da fronteira da região de estabilidade são fornecidas sob algumas suposições sobre o campo vetorial, incluindo a hiperbolicidade dos pontos de equilíbrio na fronteira da região de estabilidade e condições de transversalidade.

Neste artigo, estamos interessados em estudar caracterizações da região de estabilidade e de sua fronteira quando o sistema está sujeito a variação de parâmetros. A análise do comportamento da região de estabilidade sob variações do parâmetro (bifurcações da região de estabilidade) encontra aplicações importantes, por exemplo, na análise de colapso de tensão de sistemas elétricos de potência [7]. Sob variação de parâmetros, bifurcações locais podem ocorrer na fronteira da região de estabilidade e a suposição de hiperbolicidade dos pontos de equilíbrio pode ser violada nos pontos de bifurcações. Logo, estudar a caracterização da fronteira da região de estabilidade em pontos de bifurcações é de fundamental importância para entender como a região de estabilidade se comporta sob variação de parâmetros.

\footnotetext{
*Autor correspondente: Fabíolo Moraes Amaral.

${ }^{1}$ Departamento de Ensino, IFBA - Campus Eunápolis, 45822-000 Eunápolis, BA, Brasil. E-mail: fabiolo@ ifba.edu.br ${ }^{2}$ Departamento de Engenharia Elétrica, EESC - USP, Av. Trabalhador Sancarlense, 400, 13566-590 São Carlos, SP, Brasil. E-mail: lfcalberto@usp.br
} 
Alguns avanços nesta direção já foram obtidos e relatados na literatura. Em [1], por exemplo, uma completa caracterização da fronteira da região de estabilidade na presença de pontos de equilíbrio sela-nó do tipo-0 e uma completa caracterização de bifurcações da região de estabilidade induzida por bifurcações sela-nó do tipo-0 foram estudadas. Essas bifurcações locais selanó do tipo-0 na fronteira da região de estabilidade provocam mudanças drásticas no tamanho da região de estabilidade. Em [2], técnicas para estimar a região de estabilidade na ocorrência de bifurcações sela-nó do tipo-0, incluindo estimativas que são uniformes com relação a variação dos parâmetros foram desenvolvidas. Em [3] uma completa caracterização da fronteira da região de estabilidade na presença de pontos de equilíbrio sela-nó do tipo- $k$, com $k \geq 0$ foram apresentadas. Em [6], a caracterização da fronteira da região de estabilidade na presença de pontos de equilíbrio não-hiperbólicos do tipo Hopf foram desenvolvidas como o primeiro passo para entender o comportamento da região de estabilidade na ocorrência de bifurcações locais do tipo Hopf na fronteira da região de estabilidade.

Neste artigo, uma caracterização completa da fronteira da região de estabilidade na vizinhança de um valor de bifurcação sela-nó do tipo- $k$, com $k \geq 0$ é apresentada. Essa caracterização é o primeiro passo na busca de estimativas ótimas da região de estabilidade na ocorrência de bifurcações sela-nó do tipo- $k$, com $k \geq 0$ na fronteira da região de estabilidade.

Este artigo é organizado da seguinte maneira. Na Seção 2, uma revisão da caracterização da fronteira da região de estabilidade de sistemas dinâmicos autonômos não lineares é apresentada, incluindo a robustez da caracterização da fronteira da região de estabilidade sob as condições de hiperbolicidade e transversalidade. A principal contribuição deste artigo é apresentada na Seção 3. Mais precisamente, uma caracterização completa da fronteira da região de estabilidade na vizinhança de um valor de bifurcação sela-nó do tipo- $k, \operatorname{com} k \geq 0$ é desenvolvida. A Seção 4 é dedicada aos exemplos e a Seção 5 às considerações finais.

\section{CARACTERIZAÇÃO DA FRONTEIRA DA REGIÃO DE ESTABILIDADE}

Considere o sistema dinâmico autônomo não linear

$$
\dot{x}=f(x)
$$

onde $x \in \mathbb{R}^{n}$ e $f: \mathbb{R}^{n} \rightarrow \mathbb{R}^{n}$ é um campo vetorial de classe $C^{r}$ com $r \geq 2$. A solução de (2.1) começando em $x$ no tempo $t=0$ é denotada por $\varphi(t, x)$.

Um ponto $x^{*} \in \mathbb{R}^{n}$ é um ponto de equilíbrio de (2.1) se $f\left(x^{*}\right)=0$. Um ponto de equilíbrio $x^{*}$ de (2.1) é hiperbólico se nenhum autovalor da matriz Jacobiana $D_{x} f\left(x^{*}\right)$ tem parte real igual a zero. Um ponto de equilíbrio hiperbólico $x^{*}$ é um ponto de equilíbrio assintoticamente estável se todos os autovalores de $D f\left(x^{*}\right)$ tem parte real negativa; caso contrário é um ponto de equilíbrio instável. Um conjunto $S \subset \mathbb{R}^{n}$ é um conjunto invariante de (2.1) se toda trajetória de (2.1) começando em $S$ permanece em $S$ para todo $t \in \mathbb{R}$.

A região de estabilidade de um ponto de equilíbrio assintoticamente estável $x_{s}$ de (2.1) é definida como

$$
A\left(x_{s}\right)=\left\{x \in \mathbb{R}^{n}: \varphi(t, x) \rightarrow x_{s} \text { quando } t \rightarrow \infty\right\} .
$$


A região de establidade $A\left(x_{S}\right)$ é um conjunto invariante, aberto e difeomorfo ao $\mathbb{R}^{n}$ [4]. O fecho $\overline{A\left(x_{s}\right)}$ é invariante e a fronteira da região de estabilidade $\partial A\left(x_{s}\right)$ é um conjunto fechado e invariante [4]. Uma caracterização completa da fronteira da região de estabilidade de uma ampla classe de sistemas dinâmicos foi apresentada em [4]. Considere o sistema não linear (2.1) satisfazendo as seguintes suposições:

(A1) Todos os pontos de equilíbrios em $\partial A\left(x_{s}\right)$ são hiperbólicos.

(A2) As variedades estáveis e instáveis dos pontos de equilíbrio em $\partial A\left(x_{s}\right)$ satisfazem a condição de transversalidade.

(A3) Toda trajetória em $\partial A\left(x_{s}\right)$ se aproxima de um ponto de equilíbrio quando $t \rightarrow \infty$.

As afirmações (A1) e (A2) são propriedades genéricas de sistemas dinâmicos na forma de (2.1). Em outras palavras, elas são satisfeitas para a maioria dos sistemas dinâmicos na forma de (1) e, na prática, elas não precisam ser verificadas. Ao contrário, a afirmação (A3) não é uma propriedade genérica e tem de ser verificada. A existência de uma função energia é uma condição suficiente para a satisfação da afirmação (A3). Para mais detalhes sobre este assunto, consul$\operatorname{tar}[4]$.

Sob as afirmações (A1)-(A3), o teorema a seguir fornece uma caracterização completa da fronteira da região de estabilidade do sistema dinâmico não linear (2.1).

Teorema 2.1. [4] (Caracterização da fronteira da região de estabilidade) Seja $x_{s}$ um ponto de equilíbrio assintoticamente estável de (2.1) e $A\left(x_{s}\right)$ sua região de estabilidade. Se as afirmações (A1)-(A3) são satisfeitas, então:

$$
\partial A\left(x_{s}\right)=\bigcup_{i} W^{s}\left(x^{i}\right)
$$

onde $x^{i}, i=1,2, \ldots$ são os pontos de equilíbrio em $\partial A\left(x_{s}\right)$.

O Teorema 2.1 mostra que a fronteira da região de estabilidade é a união das variedades estáveis de todos os pontos de equilíbrio na fronteira da região de estabilidade.

Neste artigo, estamos interessados em estudar o comportamento da fronteira da região de estabilidade da seguinte classe de sistemas dinâmicos

$$
\dot{x}=f(x, \lambda)=f_{\lambda}(x)
$$

com $x \in \mathbb{R}^{n}, f: \mathbb{R}^{n} \times \mathbb{R} \longrightarrow \mathbb{R}^{n}$ de classe $C^{r}$, com $r \geq 2$, dependendo de um parâmetro $\lambda \in \mathbb{R}$. Dado um ponto de equilíbrio hiperbólico $x_{\lambda_{*}}$ do sistema (2.2) para $\lambda=\lambda_{*}$, o Teorema da Função Implícita garante que um único ponto de equilíbrio hiperbólico $x_{\lambda}$ do sistema perturbado (2.2) continua existindo, em uma vizinhança de $x_{\lambda_{*}}$, para todo $\lambda$ próximo à $\lambda_{*}$. Em outras palavras, um ponto de equilíbrio hiperbólico persiste sob pequenas variações do parâmetro $\lambda$. Além disso, usando a continuidade dos autovalores com relação aos parâmetros, podemos afirmar também que o tipo de estabilidade do ponto de equilíbrio perturbado $x_{\lambda}$ é o mesmo do ponto de equilíbrio 
$x_{\lambda_{*}}$. Em particular, se $x_{\lambda_{*}}^{s}$ é um ponto de equilíbrio hiperbólico assintoticamente estável do sistema (2.2) para $\lambda=\lambda_{*}$, então continua existindo, para valores de $\lambda$ próximos a $\lambda_{*}$, um único ponto de equilíbrio assintoticamente estável perturbado $x_{\lambda}^{s}$ próximo a $x_{\lambda_{*}}^{s}$. Consequentemente, faz sentido estudar o comportamento da região de estabilidade perturbada $A_{\lambda}\left(x_{\lambda}^{S}\right)$.

O próximo teorema estuda a persistência dos pontos de equilíbrio hiperbólicos na fronteira da região de estabilidade sob as afirmações (A1)-(A3).

Teorema 2.2. [5] (Persistência dos pontos de equilíbrio hiperbólicos na fronteira da região de estabilidade) Seja $x_{\lambda^{*}}^{s}$ um ponto de equilíbrio hiperbólico assintoticamente estável de (2.2), para $\lambda=\lambda^{*}$ e $A_{\lambda^{*}}\left(x_{\lambda^{*}}^{s}\right)$ sua região de estabilidade. Se as afirmações (A1)-(A3) são satisfeitas para todo $\lambda$ próximo a $\lambda^{*}$ e $x_{\lambda^{*}}^{i}, i=1,2, \ldots$ são os pontos de equilíbrio em $\partial A_{\lambda^{*}}\left(x_{\lambda^{*}}^{s}\right)$, então existe $\epsilon>0$ tal que, para todo $\lambda \in\left(\lambda^{*}-\epsilon, \lambda^{*}+\epsilon\right)$, os pontos de equilíbrio perturbados $x_{\lambda}^{i}$, $i=1,2, \ldots$ estão também na fronteira da região de estabilidade de $x_{\lambda}^{s}$, e

$$
\bigcup_{i} W_{\lambda}^{s}\left(x_{\lambda}^{i}\right) \subseteq \partial A_{\lambda}\left(x_{\lambda}^{s}\right)
$$

Em [1], foi apresentado uma caracterização da fronteira da região de estabilidade sob a variação de parâmetros para um caso particular de violação da afirmação (A1), isto é, quando um ponto de equilíbrio sela-nó do tipo-0 está na fronteira da região de estabilidade. Neste artigo, estudamos uma caracterização da fronteira da região de estabilidade sob a variação de parâmetros quando pontos de equilíbrio sela-nó do tipo- $k, \operatorname{com} k \geq 0$ está na fronteira da região de estabilidade. Esta caracterização é o passo inicial para entedermos como a região de estabilidade se comporta no aparecimento de bifurcãções sela-nó do tipo- $k$, com $k \geq 0$ na fronteira da região de estabilidade.

\section{CARACTERIZAÇÃO DA FRONTEIRA DA REGIÃO DE ESTABILIDADE NA VIZINHANÇA DE UM VALOR DE BIFURCAÇÃO SELA-NÓ DO TIPO- $K$}

Nesta seção, uma caracterização da fronteira da região de estabilidade na vizinhança de um valor de bifurcação sela-nó do tipo- $k$, com $k \geq 0$ é apresentada. Começamos a seção com alguns conceitos da teoria de bifurcação sela-nó.

Definição 3.1. [8] (Ponto de equilíbrio sela-nó) Um ponto de equilíbrio não hiperbólico $x_{\lambda_{0}} \in$ $\mathbb{R}^{n}$ de (2.2), para um parâmetro fixo $\lambda=\lambda_{0}$, é chamado um ponto de equilíbrio sela-nó $e$ $\left(x_{\lambda_{0}}, \lambda_{0}\right)$ um ponto de bifurcação sela-nó se as seguintes afirmações são satisfeitas:

(C1) $D_{x} f_{\lambda_{0}}\left(x_{\lambda_{0}}\right)$ tem um único autovalor simples igual a 0 com $v$ um autovetor à direita e $w \grave{a}$ esquerda.

(C2) $w\left(D_{\lambda} f\left(x_{\lambda_{0}}, \lambda_{0}\right)\right) \neq 0$.

(C3) $w\left(D_{x}^{2} f_{\lambda_{0}}\left(x_{\lambda_{0}}\right)(v, v)\right) \neq 0$. 
Observação 1. A notação $w\left(D_{x}^{2} f_{\lambda_{0}}\left(x_{\lambda_{0}}\right)(v, v)\right)$ descrita em $(C 3)$ expressa um número real que é o resultado do produto interno entre os vetores $w$ e $D_{x}^{2} f_{\lambda_{0}}\left(x_{\lambda_{0}}\right)(v, v)$. Além disso, essa propriedade garante que o campo vetorial dado em (2.2), restrito a variedade central, tem comportamento quadrático.

Um ponto de equilíbrio sela-nó ou um ponto de bifurcação sela-nó podem ser classificados em tipos de acordo com o número de autovalores de $D_{x} f_{\lambda_{0}}\left(x_{\lambda_{0}}\right)$ com parte real positiva.

Definição 3.2. (Tipo de bifurcação sela-nó) Um ponto de equilíbrio sela-nó $x_{\lambda_{0}}$ de (2.2), para um parâmetro $\lambda=\lambda_{0}$, é chamado um ponto de equilíbrio sela-nó do tipo-k e $\left(x_{\lambda_{0}}, \lambda_{0}\right)$ um ponto de bifurcação sela-nó do tipo-k se $D_{x} f_{\lambda_{0}}\left(x_{\lambda_{0}}\right)$ tem $k$ autovalores com parte real positiva e $n-k-1$ com parte real negativa.

Observação 2. O parâmetro $\lambda_{0}$ da Definição 3.2 é chamado um valor de bifurcação sela-nó do tipo- $k$.

O teorema a seguir descreve o comportamento dinâmico do sistema (2.2) próximo a um ponto de bifurcação sela-nó do tipo- $k, \operatorname{com} k \geq 0$, sua demonstração pode ser encontrada em [8].

Teorema 3.3. [8] Seja $\left(x_{\lambda_{0}}, \lambda_{0}\right)$ um ponto de bifurcação sela-nó do tipo-k, com $k \geq 0$ de (2.2). Então existe uma vizinhança $U$ de $x_{\lambda_{0}}$ e $\delta>0$ tal que, dependendo dos sinais das expressões em (C2) e (C3), não existe ponto de equilíbrio em $U$ quando $\lambda \in\left(\lambda_{0}-\delta, \lambda_{0}\right)\left[\lambda \in\left(\lambda_{0}, \lambda_{0}+\delta\right)\right] e$ existem dois pontos de equilíbrio em $U$ para cada $\lambda \in\left(\lambda_{0}, \lambda_{0}+\delta\right)\left[\lambda \in\left(\lambda_{0}-\delta, \lambda_{0}\right)\right]$. Os dois pontos de equilíbrio em $U$ são hiperbólicos do tipo-k e tipo- $(k+1)$, respectivamente. Além disso, a variedade estável do ponto de equilíbrio hiperbólico tipo-k intercepta a variedade instável do ponto de equilíbrio hiperbólico tipo- $(k+1)$ ao longo de uma variedade unidimensional.

Sejam $x_{\lambda_{0}}^{s}$ um ponto de equilíbrio assintoticamente estável de (2.2) e $A_{\lambda_{0}}\left(x_{\lambda_{0}}^{s}\right)$ sua região de estabilidade para um parâmetro fixo $\lambda=\lambda_{0}$. Considere as seguintes afirmações:

(A1') Todos os pontos de equilíbrio em $\partial A_{\lambda_{0}}\left(x_{\lambda_{0}}^{s}\right)$ são hiperbólicos ou pontos de equilíbrio selanó.

(A2' $)$ As seguintes condições de transversalidade são satisfeitas:

(i) As variedades estáveis e instáveis dos pontos de equilíbrio em $\partial A_{\lambda_{0}}\left(x_{\lambda_{0}}^{s}\right)$ satisfazem a condição de transversalidade.

(ii) As variedades instáveis dos pontos de equilíbrio e a componente estável da variedade centro-estável dos pontos de equilíbrio do tipo- $k$, com $1 \leq k \leq n-2$, na $\partial A_{\lambda_{0}}\left(x_{\lambda_{0}}^{s}\right)$ satisfazem a condição de transversalidade.

(iii) As variedades instáveis dos pontos de equilíbrio e a componente estável da variedade central dos pontos de equilíbrio sela-nó do tipo- $(n-1)$ em $\partial A_{\lambda_{0}}\left(x_{\lambda_{0}}^{s}\right)$ satisfazem a condição de transversalidade. 
(iv) As variedades estáveis dos pontos de equilíbrio e a componente instável da variedade central dos pontos de equilíbrio sela-nó do tipo-0 em $\partial A_{\lambda_{0}}\left(x_{\lambda_{0}}^{s}\right)$ satisfazem a condição de transversalidade.

(v) A componente estável das variedades centro-estáveis dos pontos de equilíbrio sela-nó do tipo- $k$, com $1 \leq k \leq n-2$, e a componente instável da variedade centro-estável dos pontos de equilíbrio sela-nó do tipo-0 em $\partial A_{\lambda_{0}}\left(x_{\lambda_{0}}^{s}\right)$ satisfazem a condição de transversalidade.

(vi) A componente estável das variedades centrais dos pontos de equilíbrio sela-nó do tipo- $(n-1)$ e a componente instável da variedade cental dos pontos de equilíbrio sela-nó do tipo-0 em $\partial A_{\lambda_{0}}\left(x_{\lambda_{0}}^{s}\right)$ satisfazem a condição de transversalidade.

As afirmações $\left(A 1^{\prime}\right)$ e $\left(A 2^{\prime}\right)$ são mais fracas que $(A 1)$ e $(A 2)$ respectivamente. Afirmação $\left(A 1^{\prime}\right)$ permite a presença de pontos de equilíbrio não-hiperbólicos sela-nó na fronteira da região de estabilidade.

O teorema a seguir estuda o comportamento da fronteira da região de estabilidade na vizinhança de um ponto de equilíbrio sela-nó do tipo- $k, \operatorname{com} k \geq 0$.

Teorema 3.4. (Comportamento da fronteira da região de estabilidade na vizinhança de um ponto de equilíbrio sela-nó do tipo- $k$, com $k \geq 0)$ : Sejam $x_{\lambda_{0}}^{s}$ um ponto de equilíbrio hiperbólico assintoticamente estável de (2.2) e $A_{\lambda_{0}}\left(x_{\lambda_{0}}^{s}\right)$ sua região de estabilidade para $\lambda=\lambda_{0}$. Se as afirmações $(A 1),(A 2)$ e (A3) são satisfeitas em um intervalo aberto contendo $\lambda_{0}$, exceto no valor de bifurcação sela-nó do tipo- $k \lambda_{0}$, com $k \geq 0$, onde as afirmações $\left(A 1^{\prime}\right),\left(A 2^{\prime}\right)$ e $(A 3)$ são satisfeitas e que as variedades instáveis de todos os pontos de equilíbrio sela-nó do tipo-r, com $r \geq 1$ na fronteira da região de estabilidade $\partial A_{\lambda_{0}}\left(x_{\lambda_{0}}^{s}\right)$, tem interseção não-vazia com o fecho da região de estabilidade $A_{\lambda_{0}}\left(x_{\lambda_{0}}^{s}\right)$, então:

(i) Se $\left(x_{\lambda_{0}}, \lambda_{0}\right)$ é um ponto de bifurcação sela-nó do tipo-0, com $x_{\lambda_{0}}$ pertencendo à fronteira da região de estabilidade $\partial A_{\lambda_{0}}\left(x_{\lambda_{0}}^{s}\right)$, então existe $\beta>0$ tal que para todo $\lambda \in\left(\lambda_{0}-\beta, \lambda_{0}\right)$ tem-se que

$$
y_{\lambda^{0}} \notin \partial A_{\lambda}\left(x_{\lambda}^{s}\right) \text { e } y_{\lambda^{1}} \in \partial A_{\lambda}\left(x_{\lambda}^{s}\right)
$$

onde $y_{\lambda^{0}}$ e $y_{\lambda^{1}}$ são os pontos de equilíbrio hiperbólicos originados da bifurcação sela-nó do tipo-0.

(ii) $S e\left(x_{\lambda_{0}}, \lambda_{0}\right)$ é um ponto de bifurcação sela-nó do tipo-r, com $r \geq 1$, com $x_{\lambda_{0}}$ pertencendo à fronteira da região de estabilidade $\partial A_{\lambda_{0}}\left(x_{\lambda_{0}}^{s}\right)$, então existe $\beta>0$ tal que para todo $\lambda \in\left(\lambda_{0}-\beta, \lambda_{0}\right)$ tem-se que

$$
y_{\lambda^{r}} \in \partial A_{\lambda}\left(x_{\lambda}^{s}\right) \text { e } y_{\lambda^{r+1}} \in \partial A_{\lambda}\left(x_{\lambda}^{s}\right)
$$

onde $y_{\lambda^{r}}$ e $y_{\lambda^{(r+1)}}$ são os pontos de equilíbrio hiperbólicos instáveis originados da bifurcação sela-nó do tipo-r, com $r \geq 1$. 
Demonstração. (i) Esse item foi demonstrado em [1]. (ii) Como $x_{\lambda_{0}} \in \partial A_{\lambda_{0}}\left(x_{\lambda_{0}}^{s}\right), W_{\lambda_{0}}^{u}\left(x_{\lambda_{0}}\right) \cap$ $\overline{A_{\lambda_{0}}\left(x_{\lambda_{0}}^{s}\right)} \neq \emptyset$ e as suposições $\left(A 1^{\prime}\right),\left(A 2^{\prime}\right)$ e $(A 3)$ são satisfeitas para $\lambda=\lambda_{0}$, podemos afirmar que $W_{\lambda_{0}}^{u}\left(x_{\lambda_{0}}\right) \cap A_{\lambda_{0}}\left(x_{\lambda_{0}}^{s}\right) \neq \varnothing$. Além disso, esta interseção é transversal. Por outro lado, como $A_{\lambda_{0}}\left(x_{\lambda_{0}}^{s}\right)=W_{\lambda_{0}}^{s}\left(x_{\lambda_{0}}^{s}\right)$, temos que $W_{\lambda_{0}}^{u}\left(x_{\lambda_{0}}\right) \cap W_{\lambda_{0}}^{s}\left(x_{\lambda_{0}}^{s}\right) \neq \emptyset$. Exlorando o fato que $W_{\lambda}^{u}\left(y_{\lambda^{r}}\right) \mathrm{e}$ $W_{\lambda}^{s}\left(x_{\lambda}^{s}\right)$ dependem continuamente de $\lambda$ podemos afirmar que existe $\beta>0$ tal que $W_{\lambda}^{u}\left(y_{\lambda^{r}}\right) \cap$ $W_{\lambda}^{s}\left(x_{\lambda}^{s}\right) \neq \varnothing$ para todo $\lambda \in\left(\lambda_{0}-\beta, \lambda_{0}\right)$, ou seja, $W_{\lambda}^{u}\left(y_{\lambda^{r}}\right) \cap A_{\lambda}\left(x_{\lambda}^{s}\right) \neq \varnothing$ para todo $\lambda \in$ $\left(\lambda_{0}-\beta, \lambda_{0}\right)$. Portanto, o Teorema 3-7 provado em [4] garante que $y_{\lambda^{r}} \in \partial A_{\lambda}\left(x_{\lambda}^{s}\right)$ para todo $\lambda \in\left(\lambda_{0}-\beta, \lambda_{0}\right)$. Mostraremos agora que $y_{\lambda^{r+1}} \in \partial A_{\lambda}\left(x_{\lambda}^{s}\right)$. Pelo Teorema 3.3 a variedade estável do ponto de equilíbrio hiperbólico tipo- $r$ intercepta a variedade instável do ponto de equilíbrio hiperbólico tipo- $(r+1)$ ao longo de uma variedade unidimensional, ou seja, $W_{\lambda}^{s}\left(y_{\lambda^{r}}\right) \cap$ $W_{\lambda}^{u}\left(y_{\lambda^{r+1}}\right) \neq \emptyset$. Como $y_{\lambda^{r}} \in \partial A_{\lambda}\left(x_{\lambda}^{s}\right)$ para todo $\lambda \in\left(\lambda_{0}-\beta, \lambda_{0}\right)$, novamente o Teorema 37 provado em [4] garante que $W_{\lambda}^{s}\left(y_{\lambda^{r}}\right) \subseteq \partial A_{\lambda}\left(x_{\lambda}^{s}\right), \operatorname{logo} W_{\lambda}^{u}\left(y_{\lambda^{r+1}}\right) \cap \partial A_{\lambda}\left(x_{\lambda}^{s}\right) \neq \emptyset$, isto é, $y_{\lambda^{r+1}} \in \partial A_{\lambda}\left(x_{\lambda}^{s}\right)$ para todo $\lambda \in\left(\lambda_{0}-\beta, \lambda_{0}\right)$ e o teorema está provado.

O Teorema 3.4 afirma que, na ocorrência de uma bifurcação sela-nó do tipo-r, com $r \geq 1$ na fronteira da região de estabilidade, necessariamente os dois pontos de equilíbrio hiperbólicos que coalescem e desaparecem na bifurcação sela-nó pertencem à fronteira da região de estabilidade. Caso contrário, a suposição genérica de transversalidade seria violada.

O corolário a seguir oferece uma caracterização completa da fronteira da região de estabilidade na vizinhança de um valor de bifurcação sela-nó do tipo- $k$, com $k \geq 0$.

Corolário 3.5. (Caracterização da fronteira da região de estabilidade na vizinhança de um parâmetro de bifurcação sela-nó do tipo- $k$, com $k \geq 0$ ): Sejam $x_{\lambda_{0}}^{s}$ um ponto de equilíbrio hiperbólico assintoticamente estável de (2.2) e $A_{\lambda_{0}}\left(x_{\lambda_{0}}^{s}\right)$ sua região de estabilidade para $\lambda=\lambda_{0}$. Se as afirmações (A1), (A2) e (A3) são satisfeitas em um intervalo aberto contendo $\lambda_{0}$, exceto no valor de bifurcação sela-nó do tipo- $k \lambda_{0}$, com $k \geq 0$ onde as afirmações $\left(A 1^{\prime}\right),\left(A 2^{\prime}\right) e(A 3)$ são satisfeitas e que as variedades instáveis de todos os pontos de equilíbrio sela-nó do tipo-r, com $r \geq 1$ na fronteira da região de estabilidade $\partial A_{\lambda_{0}}\left(x_{\lambda_{0}}^{s}\right)$, tem interseção não-vazia com o fecho da região de estabilidade $A_{\lambda_{0}}\left(x_{\lambda_{0}}^{s}\right)$, então:

(i) Para $\lambda=\lambda_{0}$ temos

$$
\partial A_{\lambda_{0}}\left(x_{\lambda_{0}}^{s}\right)=\bigcup_{i} W_{\lambda_{0}}^{s}\left(w_{\lambda_{0}}^{i}\right) \bigcup_{j} W_{\lambda_{0}}^{s}\left(p_{\lambda_{0}}^{j}\right) \bigcup_{l} W_{\lambda_{0}}^{c s^{-}}\left(z_{\lambda_{0}}^{l}\right) \bigcup_{m} W_{\lambda_{0}}^{c^{-}}\left(q_{\lambda_{0}}^{m}\right)
$$

onde $w_{\lambda_{0}}^{i}$ são os pontos de equilíbrio hiperbólicos em $\partial A_{\lambda_{0}}\left(x_{\lambda_{0}}^{s}\right), p_{\lambda_{0}}^{j}$ são os pontos de equilíbrio sela-nó do tipo-0, $z_{\lambda_{0}}^{l}$ são os pontos de equilíbrio sela-nó do tipo- $k$, com $1 \leq k \leq$ $n-2$ e $q_{\lambda_{0}}^{m}$ são os pontos de equilíbrio sela-nó do tipo- $(n-1)$ em $\partial A_{\lambda_{0}}\left(x_{\lambda_{0}}^{s}\right), i, j, l, m=$ $1,2, \ldots$.

(ii) Existe $\epsilon>0$ tal que, para todo $\lambda \in\left(\lambda_{0}-\epsilon, \lambda_{0}\right)$,

$$
\partial A_{\lambda}\left(x_{\lambda}^{s}\right)=\bigcup_{i} W_{\lambda}^{s}\left(w_{\lambda}^{i}\right) \bigcup_{j} W_{\lambda}^{s}\left(y_{\lambda^{k}}^{j}\right) \bigcup_{j} W_{\lambda}^{s}\left(y_{\lambda^{k+1}}^{j}\right)
$$


onde $w_{\lambda}^{i}$ são os pontos de equilíbrio hiperbólicos perturbados em $\partial A_{\lambda}\left(x_{\lambda}^{s}\right), y_{\lambda^{k}}^{j}$ e $y_{\lambda^{k+1}}^{j}$ são os pontos de equilíbrio hiperbólicos instáveis originados da bifurcação sela-nó do tipo-k, com $k \geq 0$, que também pertencem a $\partial A_{\lambda}\left(x_{\lambda}^{s}\right), i, j,=1,2, \ldots$.

(iii) Existe $\epsilon>0$ tal que, para todo $\lambda \in\left(\lambda_{0}, \lambda_{0}+\epsilon\right)$,

$$
\partial A_{\lambda}\left(x_{\lambda}^{s}\right)=\bigcup_{i} W_{\lambda}^{s}\left(w_{\lambda}^{i}\right)
$$

onde $w_{\lambda}^{i}$ são os pontos de equilíbrio hiperbólicos perturbados em $\partial A_{\lambda}\left(x_{\lambda}^{s}\right), i=1,2, \ldots$.

\section{EXEMPLOS}

Considere o sistema de equações diferencias

$$
\begin{aligned}
& \dot{x}=1-\lambda \operatorname{sen}(x)-2 \operatorname{sen}(x-y) \\
& \dot{y}=1-3 \operatorname{sen}(y)-2 \operatorname{sen}(y-x)
\end{aligned}
$$

onde $(x ; y ; z) \in \mathbb{R}^{3}$ e $\lambda \in \mathbb{R}$.

O sistema (4.1) possui, para $\lambda_{0}=2,84$, um ponto de equilíbrio hiperbólico assintoticamente estável $x_{\lambda_{0}}^{s}=(0,35 ; 0,35)$ e um ponto de equilíbrio sela-nó do tipo- $1 x_{\lambda_{0}}=(1,3 ; 3,4)$. O ponto de equilíbrio sela-nó do tipo-1 pertence à fronteira da região de estabilidade $\partial_{\lambda_{0}}(0,35 ; 0,35)$, ver Figura 1. Para $\lambda=2,87$, o sistema (4.1) possui um ponto de equilíbrio hiperbólico assintoticamente estável $x_{\lambda}^{s}=(0,34 ; 34), y_{\lambda^{1}}=(1,15 ; 3,33)$ um ponto de equilíbrio hiperbólico instável do tipo-1 e $y_{\lambda^{2}}=(1,48 ; 3,4)$ um ponto de equilíbrio hiperbólico instável do tipo-2. Os pontos de equilíbrio $y_{\lambda^{1}}$ e $y_{\lambda^{2}}$ são originados do ponto de equilíbrio sela-nó do tipo-1 em uma bifurcação sela-nó do tipo-1. Além disso, $y_{\lambda^{1}} \in \partial A_{\lambda}(0,34 ; 34)$ e $y_{\lambda^{2}} \in \partial A_{\lambda}(0,34 ; 34)$, de acordo com o Teorema 3.4, vide Figura 2.

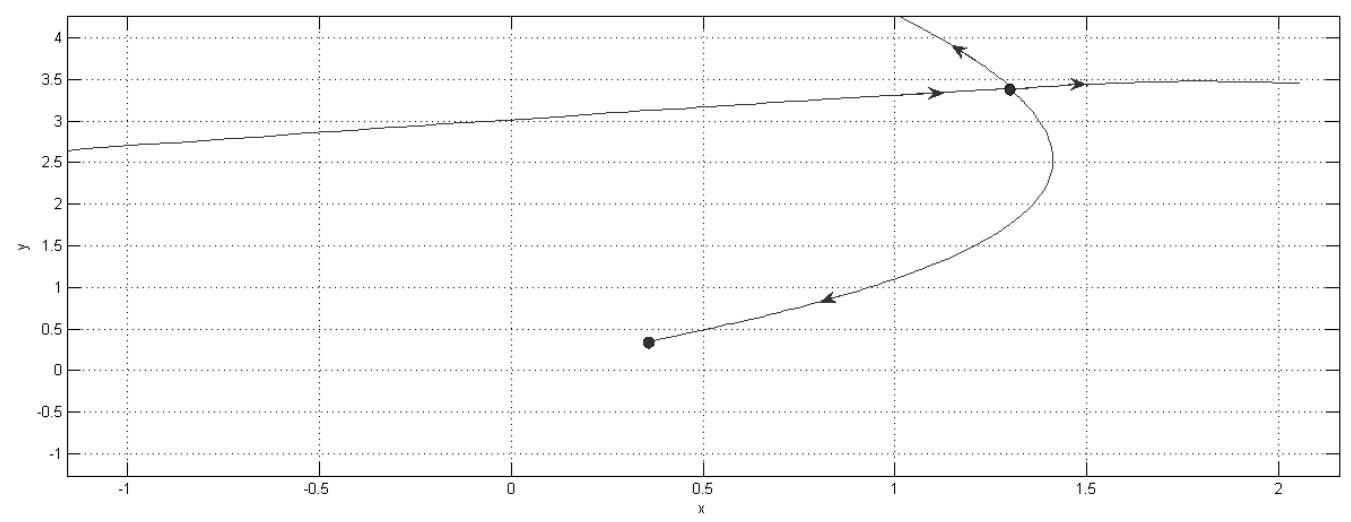

Figura 1: O ponto de equilíbrio sela-nó do tipo-1 $x_{\lambda_{0}}=(1,3 ; 3,4)$ pertence a fronteira da região de estabilidade $\partial A_{\lambda_{0}}(0,35 ; 35)$. 


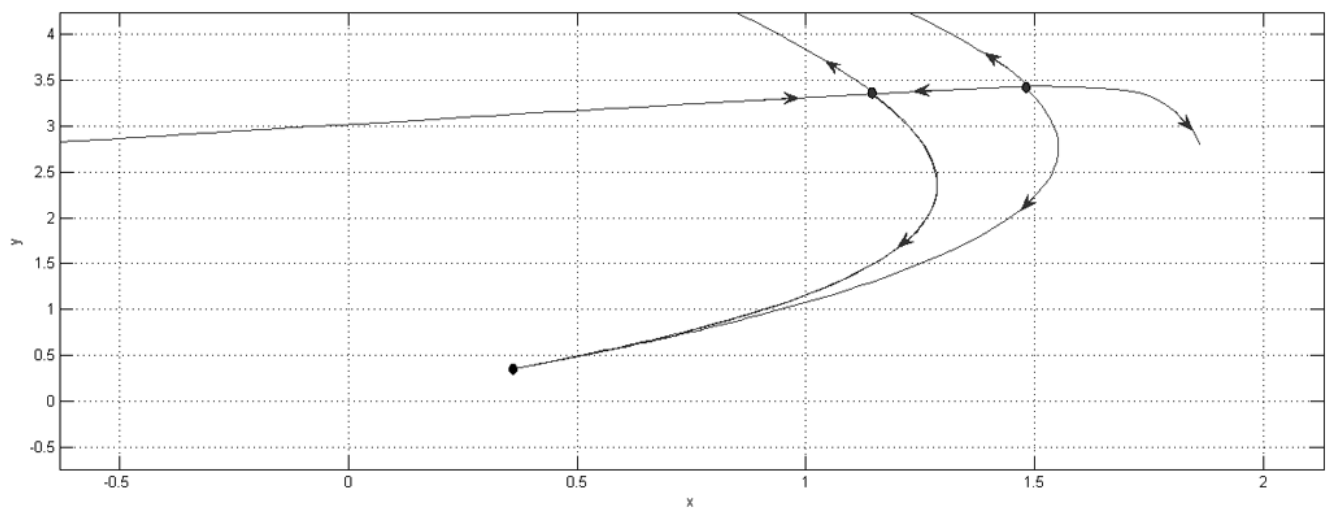

Figura 2: O ponto de equilíbrio hiperbólico do tipo-1 $(1,15 ; 3,33)$ e o ponto de equilíbrio hiperbólico do tipo- $2(1,48 ; 3,4)$ pertencem a fronteira da região de estabilidade $\partial A_{\lambda}(0,34 ; 34)$.

\section{CONSIDERAÇÕES FINAIS E TRABALHOS FUTUROS}

Neste trabalho, estudamos o comportamento da região de estabilidade de sistemas dinâmicos autônomos não-lineares sob a variação de parâmetros. Inicialmente, apresentamos um resultado que descreve o comportamento local da fronteira região de estabilidade na vizinhança de um ponto de equilíbrio sela-nó do tipo- $k$, com $k \geq 0$. Uma caracterização completa da fronteira da região de estabilidade na vizinhança de um parâmetro de bifurcação sela-nó do tipo- $k$, com $k \geq 0$ também foi apresentada. Essa caracterização generaliza os resultados existentes na literatura e demonstrados em [1]. Um exemplo para validar os resultados apresentados foi explorado. Trabalho futuros nesta área incluem a análise de outros tipos de bifurcação na fronteira da região de estabilidade, tais como bifurcações de Hopf. Aplicações promissoras desses resultados incluem a análise de estabilidade de sistemas elétricos de potência, ver [7] e a teoria de redes neurais artificiais, ver [1].

\footnotetext{
ABSTRACT. The behavior of the stability region of dynamic systems subject to parameter variation is studied in this paper. The behavior of the stability region and its boundary when the system undergoes a type- $k$ saddle-node bifurcation, with $k \geq 0$ on the stability boundary is investigated. A complete characterization of the stability boundary in the neighborhood of a type- $k$ saddle-node bifurcation value, with $k \geq 0$ is presented in this paper.
}

Keywords: stability region, stability boundary, saddle-node bifurcation.

\section{REFERÊNCIAS}

[1] F.M. Amaral \& L.F.C. Alberto. Stability Region Bifurcations of Nonlinear Autonomous Dynamical Systems: Type-Zero Saddle-Node Bifurcations. International Journal of Robust and Nonlinear Control, 21(6) (2011), 591-612. 
[2] F.M. Amaral \& L.F.C. Alberto. Type-zero saddle-node bifurcations and stability region estimation of nonlinear autonomous dynamical systems. International Journal of Bifurcation and Chaos in Applied Sciences and Engineering, 22(1) (2012), 1250020-1.

[3] F.M. Amaral, J.R.R. Gouveia Jr. \& L.F.C. Alberto. Characterization of saddle-node equilibirum points on the stability boundary of nonlinear autonomous dynamical system. Conferência Brasileira de Dinâmica, Controle e Aplicações, Anais DINCON, (2013).

[4] H.-D. Chiang, M.W. Hirsch \& F.F. Wu. Stability regions of nonlinear autonomous dynamical systems. IEEE Transactions on Automatic Control, 33 (1988), 16-27.

[5] H.-D. Chiang \& Chia-Chu. Theorical foundation of the BCU method for direct stability analysis of network-reduction power system models with small transfer conductances. IEEE Transactions on Circuits and Systems-I: Fundamental Theory and Applications, 42 (1995), 252-265.

[6] J.R.R. Gouveia Jr., F.M. Amaral \& L.F.C. Alberto. Stability boundary characterization of nonlinear autonomous dynamical systems in the presence of a supercritical Hopf equilibrium point. International Journal of Bifurcation and Chaos in Applied Sciences and Engineering, 23(12) (2014), 1350196-1.

[7] R.B. de Lima Guedes, L.F.C. Alberto \& N.G. Bretas. Power System Low-Voltage Solutions Using an Auxiliary Gradient System for Voltage Collapse Purposes. IEEE Transactions on Power Systems, 20 (2005), 1528-1537.

[8] J. Sotomayor. Generic bifurcations of dynamical systems. Dinamical Systems, (1973), 549-560.

[9] V. Venkatasubramanian, H. Schattler \& J. Zaborszky. A taxonomy of the dynamics of large differential-algebraic systems. Proceedings IEEE, 83 (1995), 1530-1561. 